\title{
Contour Extraction of Femur and Tibia Condyles on Plain Anteroposterior (AP) Radiograph
}

\author{
Humera Tariq \\ S.M. Aqil Burney \\ Department of Computer Science, University of Karachi
}

\begin{abstract}
Condyle of femur (F) and tibia (T) are key bony landmarks around the knee joint. The condyles contour extraction is the first key step towards: quantification of femorotibial (FT) joint space width (JSW), to assess FT joint space narrowing (JSN), to determine knee joint alignment angles and in defining the lateral and medial condyle radii. This paper aims to contour extraction of femur and tibia condyles on plain anteroposterior (AP) knee radiograph. A number of standard $\mathrm{OA}$ and non-OA AP radiographs in DICOM format are acquired. The images are resized and filtered to detect femoral and tibia condyles edge pixels respectively. Thresholding is then applied which introduce outliers. After removing outliers spline interpolation and smoothing is applied to trace and extract the femur and tibia contours. The results demonstrates promising extracted contours on non-OA and moderate OA radiographs but needs improvement in radiographs showing severe OA. The femur and tibia contours obtained are well localized, smooth and have no broken lines.
\end{abstract}

\section{General Terms}

Image Processing, Thresholding, Edge Detection, Contour Extraction, Prewitt Operator, Sobel Operator, Interpolation, Smoothing.

\section{Keywords}

DICOM; Knee Joint ; Osteoarthritis; Femur; Tibia; Contour; AP radiograph; Joint Space Width.

\section{INTRODUCTION}

The very first medical investigation in the diagnostic of degenerative joint disease OA, knee fracture or knee surgery is $\mathrm{x}$-ray or plain radiograph. This imaging modality is widely used not only because it is accessible and relatively inexpensive but is still serves as a gold standard in OA diagnostics and surgery. The primary reason for extraction of femur and tibia condyles on knee AP radiograph is to look for non-uniform joint space loss. This space tends to shrink as the cartilage around the bone is destroyed. Other radiographic findings may include development of bony growth, joint damaging, cyst formation, and subluxation. The patient's initial radiographs may not show all of the findings of OA disease and only minimal, non-uniform joint space narrowing may be present so joint space narrowing is the key sign for early detection of knee OA. The quantification of these visual parameters is a challenging problem in the field of biomedical imaging for diagnostics and surgical implications. In general, contour extraction follows edge detection, which is a process of identifying pixels in a digital image at which the image brightness changes sharply. The classical gradient based edge detection operators are Sobel[7], Robert[10], and Prewitt [8] each designed to be sensitive to certain types of edges. In these algorithms, the estimated gradient pixel value higher than a threshold is counted as an edge pixel. Because threshold value is often empirically determined, it is possible to lose some edges or over estimation occurs.

This paper develops an interactive algorithm to detect knee joint edge pixels which are used to extract contours of thigh bone femur and shin bone tibia using four steps. (1) Image preprocessing. (2)ROI Selection and Mask Processing. (3) Outliers Elimination. (4)Spline Interpolation and Smoothing. See Figure 1.

\begin{tabular}{|l|c|}
\hline \multicolumn{2}{|c|}{ Image Pre-Processing } \\
\hline Resizing & Subsampling \\
\hline ROI Selection \& Mask Processing \\
\hline Thresholding & Edge detection \\
\hline \multicolumn{2}{|c|}{ Outliers Elimination } \\
\hline \multicolumn{2}{|c|}{ Scanning } \\
\hline Spline Interpolation \\
\hline Curve Fitting & Moving average \\
\hline
\end{tabular}

Fig 1: Steps for Contour Extraction

The rest of the paper is organized as follows. Section 2, Review the methods available in literature for knee joint detection and boundary extraction. Section 3, discusses classical edge detection theory. Section 4, is about spline interpolation. Section 5, discusses our contour extraction strategy. Finally the conclusion is stated in section 7 followed by results and future work in Section 6 . 


\section{RELATED WORK}

Researches for automated and semi-automated digital analysis of knee radiograph have been going over [1-4] for objective quantification, diagnostic and treatment plan of OA. To detect knee joint region and the edges along knee bones is a compulsory exercise for this.

An automated approach for the boundary detection of femur and tibia was presented by Mengko[1] that first locates $400 \mathrm{x}$ 200 pixels in the center of the image then use Gaussian function and a combination of horizontal and vertical gradient image to determine the desired boundaries.

Schmidt[2] presents semi-automated digital image analysis of JSW in knee radiographs that identifies the femoral and tibial edges by first adjusting the image intensity. Then canny[9] edge detection algorithm was used to determine the distal edge of the medial and lateral femoral condyles. The user then defines the extent of the medial and lateral compartments. The inner boundary was defined as the point where the slope of the tibial spine began to increase and the outer boundary was defined as the outer edge of the tibial plateau. The cortical bone interface of the tibia was found by determining the brightest pixels in each vertical scan line. Next, the program automatically determined which of the brightest pixels was closest to the identified pixel in the previous scan line. This approach was replicated across the entire medial and lateral tibial compartment to detect femoral and tibial edges.

Oka [3] presented a fully automatic knee OA computer-aided diagnosis (KOACAD) algorithm whose image processing part involves digitizing knee radiograph as a DICOM file. The entire radiograph underwent filtering three times with a $3 \times 3$ square neighborhood median filter to reduce the image noise. Then Robert's filter was applied to extract rough outlines of tibia and femur. Then medial and lateral sides are judged by the difference of calculated widths of tibia and fibula at the level of 100 pixels above the bottom of the image. To determine the region of interest (ROI) including the tibiofemoral joint space, a vertical neighborhood difference filter was applied to identify points with high absolute values of difference of scales. The center of all the points was then calculated, and $480 \times 200$ pixels of a rectangle with the center were decided as the ROI. Within the ROI, the outline of femoral condyle was designated as the upper rim of the joint space by vertical filtering with the $3 \times 3$ square neighborhood difference filter. The two ends were determined using a canny's filter to remove the noise of the lines, and vertical lines from the ends were designated as the outside rims of the joint space. Outlines of anterior and posterior margins of the tibial plateau ware drawn similarly to that of the femoral condyle, and the middle line between the two outlines was designated as the lower rim of the joint space.

A newer joint detection algorithm on Knee X-Ray was presented in the literature by Shamir[4] that is used to find the joint and separate it from the rest of the image. This is done by using a fixed set of 20 preselected images, such that each image is a $150 \times 150$ window of a center of a joint. Finding the joint in a given knee X-ray image is performed by first downscaling the image by a factor of 10 and then scanning the image with a $15 \times 15$ shifted window. For each position, the Euclidean distance between the $15 \times 15$ pixels of the shifted window and each of the $2015 \times 15$ predefined joint images are computed. After scanning the entire possible positions, the window that recorded the smallest Euclidean distance is determined as the center of the joint and the $250 \times 200$ pixels around this center from an image is used for the automated analysis.

M.Seise[5], conducted a study for automatic segmentation of tibia and femur on knee X-ray images. He proposes a double contour active shape model in order to simultaneously segment anterior and posterior contours of the tibial plateau.

An Adapt edge detection algorithm for human knee osteoarthritis images is also proposed by Zahurul[6] that is a modification to classical edge detection Sobel operator.

\section{Edge Detection}

Pixel edges are associated with abrupt intensity changes, hence edge detection is the process to identify and locate such sharp intensity contrasts (i.e., discontinuities) in an image. Classical edge detection operators Sobel and Prewitt uses $3 \times 3$ kernels which are convolved with the original image to calculate approximations of the derivatives - one for horizontal changes, and one for vertical. If we define $\mathbf{I}$ as the source image, and $\mathbf{G}_{x}$ and $\mathbf{G}_{y}$ are two images which at each point contain the horizontal and vertical derivative approximations using Sobel Operator, the computations are as follows:

$$
G_{x}=\left[\begin{array}{lll}
-1 & 0 & 1 \\
-2 & 0 & 2 \\
-1 & 0 & 1
\end{array}\right] * I ; G_{y=}\left[\begin{array}{ccc}
-1 & -2 & -1 \\
0 & 0 & 0 \\
1 & 2 & 1
\end{array}\right] * I
$$

Where * here denotes the 2-dimensional convolution operation. If Prewitt $3 \times 3$ kernels is used, the computations are as follows:

$$
G_{x}=\left[\begin{array}{lll}
-1 & 0 & 1 \\
-1 & 0 & 1 \\
-1 & 0 & 1
\end{array}\right] * I ; G_{y=}\left[\begin{array}{ccc}
-1 & -1 & -1 \\
0 & 0 & 0 \\
1 & 1 & 1
\end{array}\right] * I
$$

The $x$-coordinate is here defined as increasing in the "right"direction, and the $y$-coordinate is defined as increasing in the "down"-direction. At each point in the image, the resulting gradient approximations can be combined to give the gradient magnitude and direction as follows:

$G=\sqrt{G_{x}^{2}+G_{y}^{2}}$ and $\theta=\tan ^{-1} G_{y} / G_{x}$

The results of popular gradient based Sobel and Prewitt Operator are shown in Fig.2.

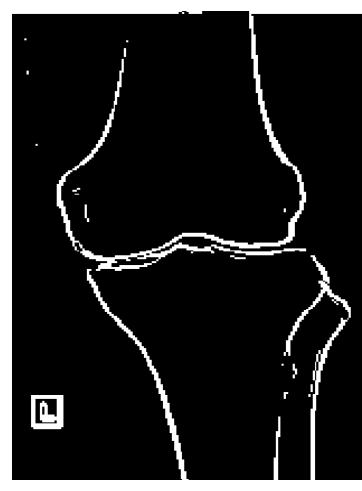

Fig 2:(a)Sobel Edges

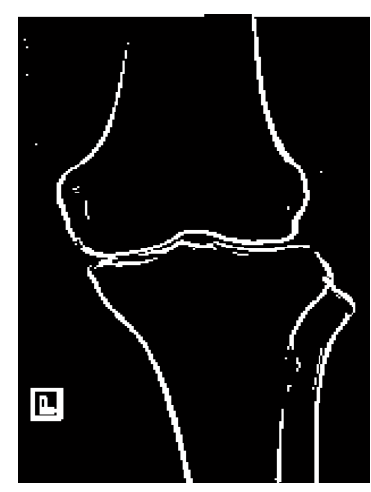

(b)Prewitt Edges 
Both the Sobel- and Prewitt-masked filters performed very well. Each generates imagewith clearly-defined edges; in fact, it is pretty much impossible to tell which filtergenerates which image when objective is to look for only horizontal and vertical edges.

\section{Spline Interpolation}

Consider a collection of known points $\left(\mathrm{t}_{0}, \mathrm{y}_{0}\right),\left(\mathrm{t}_{1}, \mathrm{y}_{1}\right),\left(\mathrm{t}_{2}, \mathrm{y}_{2}\right)$, $\left(\mathrm{t}_{3}, \mathrm{y}_{3}\right) \ldots \ldots \ldots \ldots\left(\mathrm{t}_{\mathrm{n}-1}, \mathrm{y}_{\mathrm{n}-1}\right),\left(\mathrm{t}_{\mathrm{n}}, \mathrm{y}_{\mathrm{n}}\right)$. To interpolate between these data points using traditional cubic splines, a third degree polynomial is constructed between each point. The equation to the left of point $\left(t_{i}, y_{i}\right)$ is indicated as $f_{i}$ with a $y$ value of $f_{i}\left(t_{i}\right)$ at point $t_{i}$. Similarly, the equation to the right of point $\left(t_{i}\right.$, $\left.y_{i}\right)$ is indicated as $f_{i+1}$ with a y value of $f_{i+1}\left(t_{i}\right)$ at point $t_{i}$. See Fig

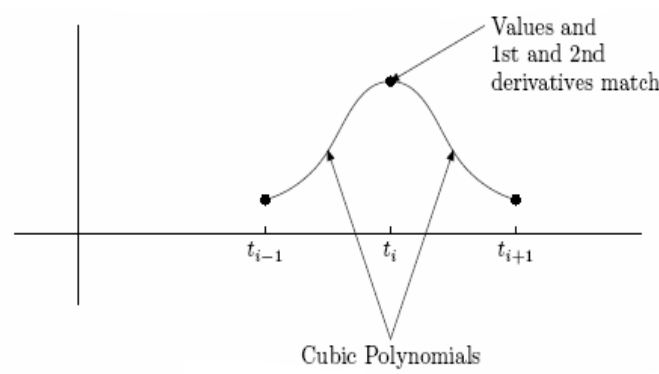

Fig 2: Interpolation using Splines

Traditionally the cubic spline function, $\mathrm{f}_{\mathrm{i}}$, is constructed based on the following criteria:

- Curves are third order polynomials,

$f_{i}(t)=a_{i}+b_{i} t+c_{i} t^{2}+d_{i} t^{3}$

- Curves pass through all the known points, $f_{i}\left(t_{i}\right)=f_{i+1}\left(t_{i+1}\right)=y_{i}$

- The slope, or first order derivative, is the same for both functions on either side of a point,

$f_{i}^{\prime}\left(t_{i}\right)=f_{i+1}^{\prime}\left(t_{i}\right)$

- The second order derivative is the same for both functions on either side of a point,

$f_{i}^{\prime \prime}\left(t_{i}\right)=f_{i+1}^{\prime \prime}\left(t_{i}\right)$

This results in a matrix of $n-1$ equations and $n+1$ unknown. The two remaining equations are based on the border conditions for the starting point, $\mathrm{f} 1\left(\mathrm{t}_{0}\right)$, and end point, $f\left(t_{n}\right)$. Historically one of the following border conditions have been used:

- Natural splines. The second order derivative of the natural splines at the end points is zero, $f_{1}^{\prime \prime}\left(t_{0}\right)=f_{n}^{\prime \prime}\left(t_{n}\right)=0$

(5a)

- Parabolic runout splines.The second order derivative of the splines at the end points is the same as at the adjacent points. The result is that the curve becomes a parabolic curve at the end points,

$f_{n}^{\prime \prime}\left(t_{n}\right)=f_{n}^{\prime \prime}\left(t_{n-1}\right)(\mathbf{5 b})$

$$
f_{1}^{\prime \prime}\left(t_{0}\right)=f_{1}^{\prime \prime}\left(t_{1}\right)
$$

- Cubic runout splines. The curve degrades to a single cubic curve over the last two intervals by setting the second order derivative of the splines at the end points to:

$$
\begin{array}{r}
f_{1}^{\prime \prime}\left(t_{0}\right)=2 f_{1}^{\prime \prime}\left(t_{1}\right)-f_{2}^{\prime \prime}\left(t_{2}\right) \\
f_{1}^{\prime \prime}\left(t_{n}\right)=2 f_{1}^{\prime \prime}\left(t_{n-1}\right)-f_{n-1}^{\prime \prime}\left(t_{n-2}\right)(\mathbf{5 c})
\end{array}
$$

- Clamped spline. The first order derivatives of the splines at the end points are set to known values

$$
f_{n}^{\prime}\left(t_{n}\right)=f^{\prime}\left(t_{n}\right)(\mathbf{5 d})
$$

$$
f_{1}^{\prime}\left(t_{0}\right)=f^{\prime}\left(t_{0}\right)
$$

In traditional cubic splines equations 2 to 5 are combined and the $n+1$ by $n+1$ tridiagonal matrix is solved to yield the cubic spline equations for each segment.As both the first and second order derivative for connecting functions are the same at every point, the result is a very smooth curve.

Even though traditional cubic splines are well behaved it does not prevent overshoot at some points due to false edge pixels. This is illustrated in Figures 1 and 2, where a natural cubic spline is fitted to sample edge pixels of femur and tibia respectively. To overcome this problem we apply smoothing after doing spline interpolation.
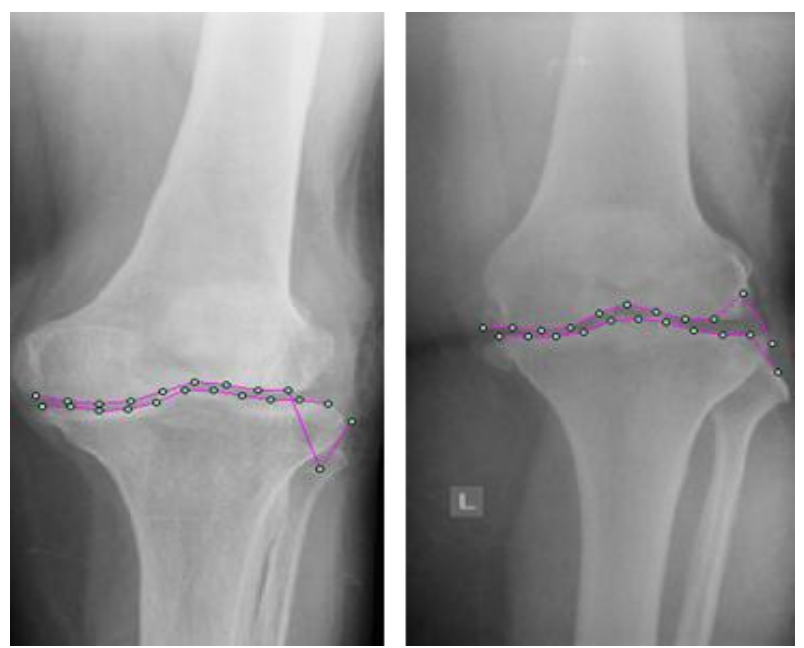

Fig 3: Spline Interpolation

\section{METHOD DESCRIPTION}

To extract the femorotibial condyle contours our methodology suggests following steps- Image preprocessing, ROI Selection and processing, Outliers Elimination, Spline interpolation and Smoothing.

\subsection{Image Preprocessing}

The data consists of medical radiographs taken from Dr. Essa's Laboratory and Diagnostic Centre. The images taken are the DICOM (Digital Imaging and Communications in Medicine) Standard radiographs at a high resolution (typical $2000 \times 3000$ pixels). These radiographs are down-scaled to a typical resolution of $256 \times 256$ pixels (8-bit) for computational reasons. Subsampling is conducted using the MATLAB im resize routine, with an 11-by-11 lowpass filter and bicubic interpolation to preserve fine details. Fig 5(a) shows the original image while Fig5(b) shows image after resizing with bicubic interpolation.

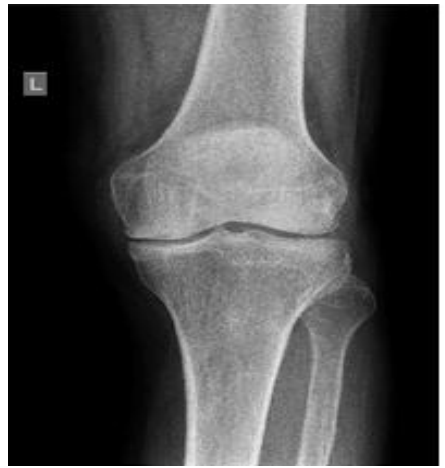

Fig 4:(a) Resizing

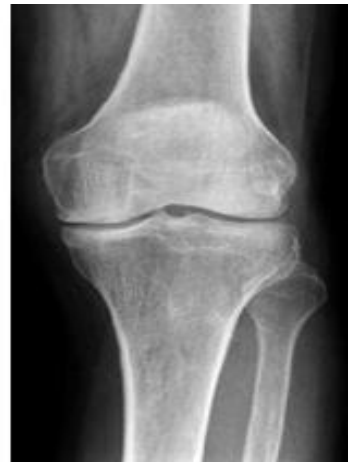

(b) Subsampling 


\subsection{ROI Selection and Mask Processing}

We interactively select the knee joint, the region of interest (ROI) including femoral and tibial condyles as shown in Fig 6(a). A mask for the knee joint portion of the image is created as shown in Fig 6(b).
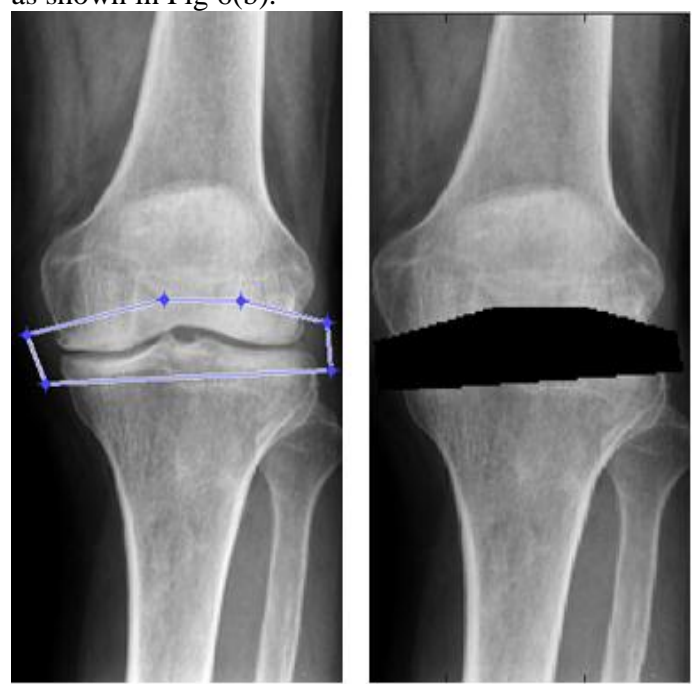

Fig 5:(a) ROI Selection

(b) Mask Processing

The masked image is processed once by prewitt[8] horizontal filter to obtain femoral condyle pixels. The same masked image is processed again by prewitt[8] vertical filter to obtain the edge pixels of tibial condyles. The two masked images with femoral and tibial edge pixels have named as femoral masked image, see Fig6(a) and tibial masked image, see Fig6(b).The Prewitt vertical difference operator and its mirror version to detect condyle pixels are:

\begin{tabular}{|l|l|l|}
\hline 1 & 1 & 1 \\
\hline 0 & 0 & 0 \\
\hline-1 & -1 & -1 \\
\hline
\end{tabular}

\begin{tabular}{|l|l|l|}
\hline-1 & -1 & -1 \\
\hline 0 & 0 & 0 \\
\hline 1 & 1 & 1 \\
\hline \multicolumn{3}{|l}{ Mirror Prewitt Operator }
\end{tabular}

The intensity of these edge pixels is set to maximum by thresholding but this introduces many false edge pixels called outliers above and below the true contours that needs to be removed. The indices of these threshold pixels are extracted to locate for condyler data. The final ROI processed image along with outliers is shown in Fig 8(a).
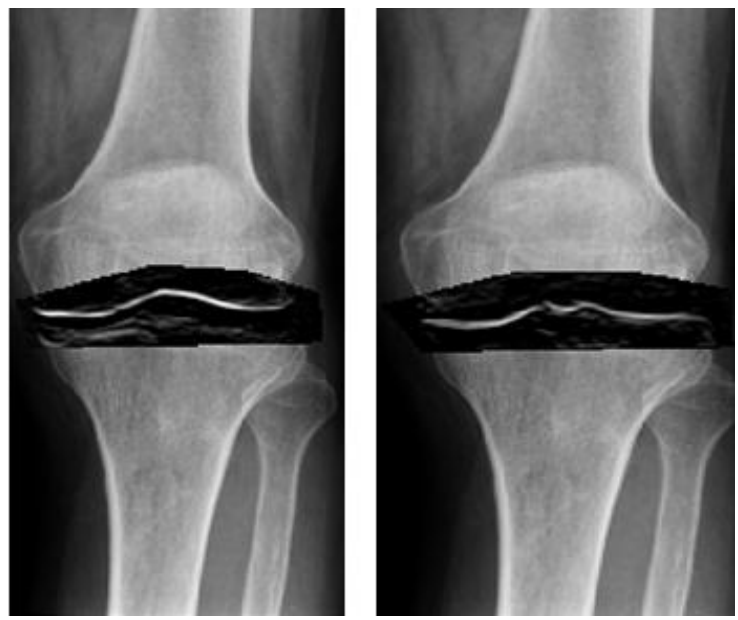

Figure 6: (a) Femoral Masked

(b) Tibial Masked

\subsection{Outliers Elimination}

The processed ROI image in Fig8(a) shows that most outliers are located above and below the vicinity of true contours and thus needs elimination.

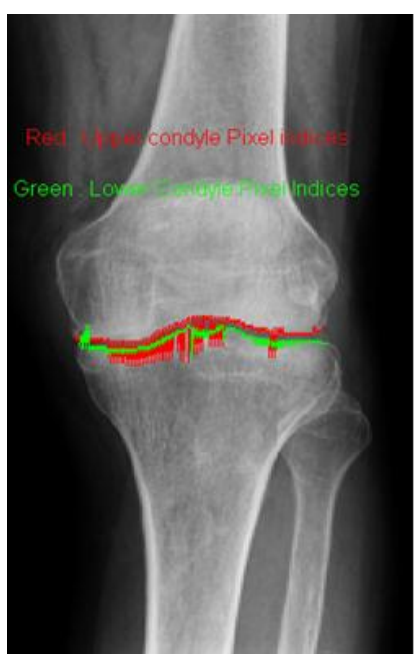

Fig 7: (a) Outlier Pixels

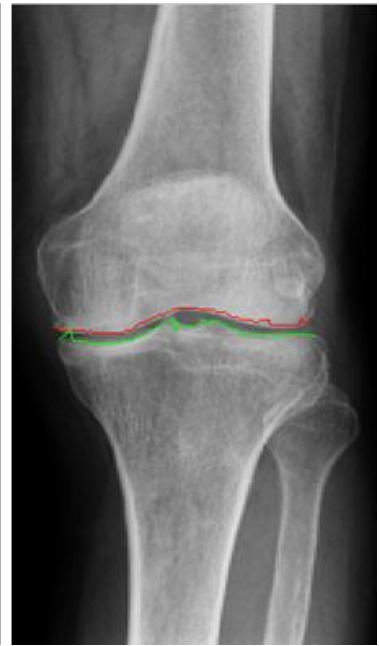

(b) Outlier Elimination
The outliers are removed by retrieving all the y components that corresponds to same $\mathrm{x}$ position and then by taking its mean. The result of outlier elimination is shown in Fig 8(b).

\subsection{Spline Interpolation and Smoothing}

Outlier elimination gives us the contours that seem very close to condyler curves but obtained contours are not smooth neither the contour points areplaced at regular intervals. So we use spline interpolation and smoothing to get equally spaced data points on final condyler contours.

\section{Results and Future Work}

We have implemented the proposed methodology using matlab and tested it on many OA and non- OA radiographs. For Result See Fig 9. The contours in non-OA and moderate OA knee radiographs are quite well localized as compared to the contours in severe OA knees radiograph. Experimental results show that the method is feasible to radiograph images and deserves further research. Future work incorporates tibial spine management in the middle of tibial contour, the completion of bone geometry using these extracted contours and to perform the reliable objective quantification of visual parameters to aid diagnostic and treatment of knee OA. The method can also be tested to other types of medical investigations like CT and MRI that have more visual clues for treatment planning.

\section{Conclusion}

In this paper, we implement a practical four-stage method that integrates edge detection with interpolation, and applied it to extract contours on Knee AP radiograph. The basic edge detection was done by classical Prewitt Operator and its mirror version.Other fundamental techniques include ROI processing,masking, Edge detection, Thresholding, Interpolation and Smoothing that are applied to successfully extract the femur and tibial condyler contours. The contours obtained are well localized, well connected and smooth. The method can be investigated for further improvement on severe osteoarthritic knee images and other medical modalities like $\mathrm{CT}$ and MRI. 

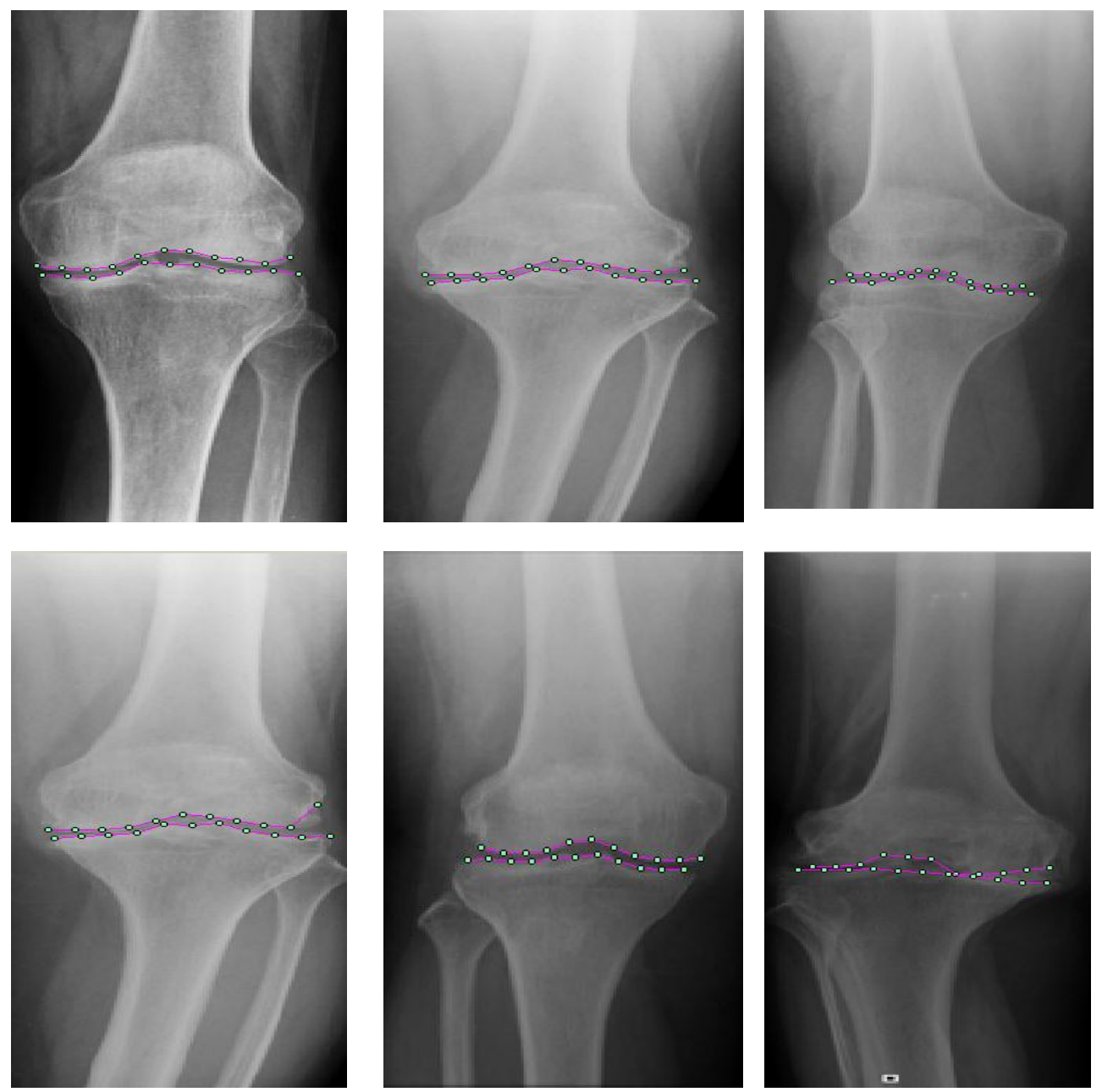

Fig 9: Results Showing Smoothed Femur and Tibial Contour

\section{ACKNOWLEDGMENTS}

We would like to thanks medical experts Dr. Arshadullah Khan, Dr. MahmoodAskari and Dr. MohsinAzam for their valuable time and helpand especially to Essa lab that provide us the images to work on.

\section{REFERENCES}

[1] Mengko TL, Wachjudi RG, Suksmono AB, Danudirdjo D. Automated detection of unimpaired joint space for knee osteoarthritis assessment. Proc 7th Intl Workshop on Enterprise Networking and Computing in Healthcare Industry. 2005:400-403.

[2] Schmidt JE, Amrami KK, Manduca A, Kaufman KR. Semi-automated digital image analysis of joint space width in knee radiographs. Skeletal Radiol 2005;639643.

[3] H.Oka, S.Muraki, T.Akune, A.Mabuchi, T.Suzuki, H.Yoshida,S.Yamamoto, K.Nakamura, N.Yoshimura and H.Kawaguchi. Fully automatic quantification of knee osteoarthritis severity on plain radiographs. Osteoarthr. Cartil., vol. 16, pp. 1300-1306, Nov. 2008.

[4] Shamir, L., Ling, S.M., Scott, W., Boss, A., Orlov, N., Macura, T., Eckley, D.M, Ferrucci, L., Goldberg, I.G.; Knee X-ray image analysis method for automated detection of Osteoarthritis, IEEE Transactions on Biomedical Engineering, vol. 56, no. 2, pp. 407-415, 2009.
[5] Matthias Seise, Stephen J.McKenna, Ian W.Ricketts and Carlos A. Wigderowitz. Segmenting Tibia and Femur from Knee X-ray Images. Med. Image Underst. Anal., pp. 103-106, 2005

[6] Syed Zahurul, Syed Zahidul and Razali Jidin. An Adapt Edge Detection Algorithm for Human Knee osteoarthritis Images.2010 International Conference on Signal Acquisition and Processing, 2010.

[7] Sobel, Camera Models and Machine Perception Stanford $\mathrm{Al}$, 1970, Memo 121.

[8] J.M.S.Prewitt. Object Enhancement and Extraction in B.S. Lipkin and A Rosenfeld eds. Picture Processing and Psychohistories, 1970.

[9] Canny J.A Computation Approach to Edge Detection [J].IEEE-PAML， 1986, 8: 679-698.

[10] L. G. Roberts. "Machine perception of 3-D solids" ser. Optical and Electro-Optical Information Processing, MIT Press, 1965.

[11] Rafael C. Gonzales, Richard E. Woods, Digital Image Processing, second edition,Prentice Hall, 2002. 